\title{
Traduire
}

Revue française de la traduction

$243 \mid 2020$

Les arts du spectacle

\section{Ionesco auteur et inspirateur. Regards croisés sur la traduction-adaptation en roumain de la pièce de théâtre Qui est ce Ionesco?, de Richard Letendre}

Adriana Apostol, Diana Lefter et Bogdan Cioaba

\section{OpenEdition}

Journals

Édition électronique

URL : http://journals.openedition.org/traduire/2147

DOI : 10.4000/traduire.2147

ISSN : 2272-9992

Éditeur

Société française des traducteurs

Édition imprimée

Date de publication : 15 décembre 2020

Pagination : 53-58

ISSN : 0395-773X

Référence électronique

Adriana Apostol, Diana Lefter et Bogdan Cioaba, « Ionesco auteur et inspirateur. Regards croisés sur la traduction-adaptation en roumain de la pièce de théâtre Qui est ce lonesco ?, de Richard Letendre »,

Traduire [En ligne], 243 | 2020, mis en ligne le 15 décembre 2020, consulté le 31 décembre 2020. URL :

http://journals.openedition.org/traduire/2147 ; DOI : https://doi.org/10.4000/traduire.2147 


\section{Ionesco auteur et inspirateur}

Regards croisés sur la traductionadaptation en roumain de la pièce de théâtre Qui est ce lonesco?, de Richard Letendre

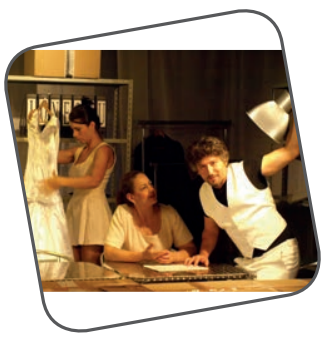

\section{Adriana Apostol, Diana Lefter, Bogdan Cioaba}

\section{Parole dite ou jouée?}

Texte ou représentation scénique, le théâtre est, indéniablement, un art qui fait de la parole sa matière première. Écrite ou dite sur la scène, cette parole doit «fonctionner» dans une égale mesure lors de la lecture ou dans l'actio scénique. De même, la traduction du texte dramatique n'engage pas seulement un savoir-lire et -interpréter le texte, mais aussi un savoir-imaginer le texte dit sur scène. Si l'on est d'accord qu'en règle générale le traducteur doit être un «lecteur idéal» du texte qu'il va rendre dans la langue cible, celui du texte dramatique peut toutefois l'être moins, surtout s'il a, dans son intention première, l'utilité et l'utilisation scénique du texte traduit.

Cette importance accordée à la parole théâtrale est l'un des éléments définitoires pour la pièce Qui est ce lonesco?, du Québécois Richard Letendre. La pièce en français est construite sur l'intertextualité des pièces ionesciennes autant 
pour le sujet et les personnages qu'au niveau textuel, plus précisément en matière de techniques de création linguistique et discursive à effets humoristiques. La succession des répliques suit la logique des sonorités des jeux de mots (que la logique conformiste ne connaît pas!). Les figures de continuité phonique (paronomases, homéotéleutes) et les répétitions parfois grammaticalement polymorphes (polyptotes, antanaclases) sont autant de procédés de création à la manière de lonesco auxquels recourt le plus souvent Letendre et que le traducteur-metteur en scène a essayé de $(r e) c r e ́ e r$ afin d'obtenir les mêmes effets sonores dans la version roumaine.

Le texte de Letendre est une actualisation de sa passion pour lonesco: fascination pour les jeux de mots, les répétitions et surtout les "sons que faisaient les mots» (entretien avec Marc-Marinescu Constantin) dans les pièces de l'auteur roumain. Ainsi, il propose un absurde typique de ce dernier, bien tempéré par l'humour. Si l'on est d'accord avec Michel Vaïs qui affirme que «[...] en empruntant à la pièce de Ionesco dialogues, personnages et situations, l'auteur aurait pu verser dans la démonstration», il faut aussi ajouter que Letendre propose un texte qui ne manque pas d'originalité et de force dramatique, exposant constamment "l'étonnement devant le fonctionnement de la langue française».

Le texte de Letendre est construit sur une double logique: l'attente-anticipation de la mort/de la fin, et l'enquête policière, en quelque sorte œdipienne - recherche du père, des racines, du début de l'existence, quête de soi-même. Une circonscription textuelle entre le début et la fin, évidente dans le texte français et bien conservée dans la version en roumain, qui respecte la même structure, véhicule la même thématique, reprend et ponctue des éléments-clés du texte source, lesquels créent cette idée d'une existence découverte à la suite d'une (en)quête dont on connaît, paradoxalement, non pas seulement le début, mais aussi la fin.

L'investigation est menée par un certain Choubert/Chouvert métamorphosé ensuite dans l'inspecteur Mallot. Certes, il s'agit du Choubert ionescien, véritable angoissé à la recherche des raisons de son existence et qui devient ici un personnage en quête de son auteur. Dans le texte français, Letendre met à profit un jeu phonétique emprunté à lonesco: le nom 
propre "Choubert» devient «Chou-vert», par le remplacement $d u$ phonème «b»par le phonème «v». Cela crée un effet humoristique et n'est pas sans rappeler, avec dérision, l'appellatif affectif «mon chou» que La Vieille des Chaises ionesciennes utilise fréquemment pour s'adresser à son mari. Dans la version roumaine, le jeu phonétique est gardé, aussi bien que la démarche logique: le remplacement d'un phonème débouche sur une autre unité sémantique: "Choubert / Somer» [ $u b \varepsilon: R]$ / [ $\left.\int \circ m \varepsilon: R\right]$; on perd toutefois l'affectivité, remplacée de manière très intéressante par l'idée d'existence vaine, sans but (Șomer, en roumain, signifie "chômeur»). Voilà un bon exemple démontrant que la fidélité à un texte source en utilisant surtout les jeux de mots et les figures de diction est difficile à obtenir, puisqu'il est peu probable qu'un équivalent avec la même force sémantique et phonique soit trouvé dans la traduction; la solution adoptée est donc, dans la plupart des cas, orientée vers la conservation de l'idée générale du texte et du style de celui-ci.

L'objet de l'(en)quête est un homme/auteur dont le nom se construit, par la technique de l'anagramme, au début de la pièce: IONESCO. Cette anagramme n'est pas construite au hasard, puisqu'on la découvre au fur et à mesure, en même temps que les mots qui constituent autant d'indices: NU, le titre du plus important ouvrage écrit par lonesco en roumain; COIN, renvoyant aux angoisses du dramaturge; NOCES, évoquant le mariage difficile des parents; ICONES, qui peut être interprété comme une anticipation des personnages emblématiques ionesciens. Dans la version roumaine, la succession est CU (avec), CUI (clou / à qui?), SUIE (il monte), SECUI (population d'origine hongroise habitant au sud-est de la Transylvanie), SONICE (soniques). II est évident que la démarche traductive ne se concentre pas sur le sémantisme des mots, mais sur l'aspect graphique et, surtout, sur la diction, confirmant encore une fois que le but du traducteur a été de se plier au style du texte et à la visée pragmatique des jeux de mots et de sonorités.

\section{Parole conservée ou transcrite?}

Le texte de Letendre a été mis en scène pour la première fois à Montréal, dans un petit espace-laboratoire, une ancienne 
boutique transformée en théâtre. L'idée d'une version textuelle et scénique en roumain est apparue à la suite d'une représentation de ce spectacle en français et a pu être réalisée en accord avec l'auteur: un texte écrit à la manière d'Eugène lonesco et susceptible de susciter l'intérêt des lecteurs et du public roumain, puisqu'il racontait "autrement» la vie compliquée et les angoisses du dramaturge franco-roumain, à travers ses personnages dramatiques. Le spectacle en roumain a d'abord été présenté à Pitești, au Théâtre Alexandru Davila où le succès fut immédiat. Suivirent des représentations au Festival international du théâtre de studio de Pitești, en 2012, puis à Strumica (Macédoine) et à Vratza (Bulgarie).

La dimension sonore du texte théâtral dit sur scène a été le point central du processus traductif avec, en arrière-pensée, le spectacle à mettre en scène. La traduction n'a pas été faite pour le seul plaisir du transcodage, elle était sous-tendue par les intentions du metteur en scène, lequel «entendait» déjà les paroles que les acteurs allaient dire sur scène. Cette "suraudition» a fonctionné comme un correcteur, car, si les mots traduits "n'épousaient pas la bouche des acteurs», c'était le signe d'un mauvais choix dans la langue cible.

Le traducteur a donc adopté une approche "pragmaticodéictique», du type: "X, ça sert à ça» (Ladmiral), la version en roumain étant notamment destinée à la mise en scène de la pièce pour un public roumain. Seulement, pendant les répétitions, les répliques des acteurs ont transformé le texte écrit/lu en texte dit/entendu, ce qui a entraîné des interventions dans le texte roumain. C'est ainsi que l'on peut parler d'un rapport dynamique bidirectionnel entre traduction et mise en scène. Un exemple (limite) en est l'énumération des synonymes du mot «policier» dans la version roumaine: Polițai, curcan, caraliu, gabor, vardist, organ, caschetar, sticlete... (Tableau VII: Le téléphone). Cette énumération ne figure pas dans le texte en français, elle est le résultat du jeu des répliques des acteurs pendant les répétitions, que le traducteur a intégré.

Dans certains tableaux, la progression du dialogue semble préférer une figure de style en particulier: la paronomase dans le Tableau III ou la polyptote dans le Tableau XVI. La version roumaine va dans le même esprit de jeux sur les homophones ou la succession de plusieurs formes grammaticales 
d'un même mot. Parfois, l'étymologie latine commune de certains mots en français et en roumain offre des solutions de traduction directe de ces figures de style: "Je suis secrétaire, je secrétais et je meurs secrètement!» devient en roumain Eu sunt secretará, am secretat ši mor secretos!

D'autre part, la complexité de la mise en équivalence de tels procédés implique des gains et des pertes. Prenons l'exemple de l'antanaclase reposant sur le jeu des homonymes «bonne»(adj.) et «bonne»(n.) qui est utilisée à plusieurs reprises dans les dialogues de la version française (Tableau IV). En roumain, il n'y a pas de paire homonymique équivalente au couple français, car l'adjectif présente un changement phonétique (buná) par rapport au nom désignant le métier (boná). Comme le texte source abonde en paronomases, la solution proposée en roumain pour cette antanaclase in absentia (Rabatel, 2015) repose sur le rapprochement phonétique des deux mots, mais également sur une certaine connotation grivoise de l'adjectif buná dans un emploi argotique. De plus, la lecture à double sens est suscitée dans la version roumaine par l'ajout explicite de E boná («Elle est bonne») au lieu de la traduction littérale de «oui»par l'équivalent roumain da. C'est ainsi que l'effet de double sens et d'ambiguïté introduit par l'antanaclase dans la pièce en français a été récupéré par un ajout et par le jeu de la paronomase in praesentia en roumain.

La version roumaine part donc d'une prémisse: c'est un texte à être dit, représenté sur scène. Ainsi, la manière de traduire le texte devient l'un des instruments aboutissant à une mise en scène originale et personnelle, ainsi qu'à l'unicité de la représentation: le souci de fidélité au texte de départ a parfois été abandonné en faveur du respect de la vision artistique du metteur en scène.

silvadius@yahoo.com bogdan_cioaba@yahoo.com diana_lefter@hotmail.com 
Silvia Adriana Apostol est maître-assistante dr. à I'université de Pitesti (Roumanie). Ses domaines d'intérêt sont le fantastique littéraire et la traduction. Traductrice depuis 2006, elle est membre du Centre de recherches sur I'imaginaire IMAGINES (Roumanie) et de la Société des Amis de Marcel Aymé (France).

Bogdan Cioabá est maître-assistant dr., à l'université de Pitesti (Roumanie) et metteur en scène au théâtre Alexandru Davila de Pitesti. Docteur en arts du spectacle, il a par ailleurs traduit plusieurs textes dramatiques du français au roumain. II est également directeur du Festival international du théâtre de studio et des formes nouvelles, organisé à Pitesti.

Diana-Adriana Lefter est maître de conférences HDR à I'université de Pitesti. Depuis 2016, elle dirige des thèses de doctorat en littérature française. Ses domaines d'intérêt sont le texte dramatique, la mythocritique et la littérature des Lumières. Elle est membre du Centre IMAGINES et de la Société internationale d'étude du XVIII ${ }^{\mathrm{e}}$ siècle.

LADMIRAL Jean-René, «Lever de rideau théorique: quelques esquisses conceptuelles» in Palimpsestes [En ligne], 16, 2004, http://journals.openedition.org/ palimpsestes/1587, consulté le 09/11/2020.

LETENDRE Richard, Qui est ce Ionesco? (texte en français et traduction en roumain par CIOABÁ Bogdan, Cine e acest Ionesco?), Éditions Nemesis Montréal (Canada), 2014.

LETENDRE Richard, Entretien avec Marc-Marinescu Constantin en ligne sur www. vimeo.com/112978387, consulté le 09/11/2020.

RABATEL Alain, «La plurisémie dans les syllepses et les antanaclases » in Vox Romanica, Francke/Narr, 74, 2015, p. 124-156, https://hal.archives-ouvertes.fr/hal01312636/document, consulté le 09/112020.

VAÏS Michel, «L'esprit est là "Qui est ce Ionesco" » in Jeu. Revue de théâtre - Théâtres de la folie, 140 (3), 2011, p. 16-17. 